Please do not remove this page

RMIT

UNIVERSITY

\title{
Two-step crystallization kinetics in colloidal hard-sphere systems
}

Schoepe, Hans-Jaochim; Bryant, Gary; Van Megen, William

https://researchrepository.rmit.edu.au/esploro/outputs/9921862041801341/filesAndLinks?institution=61RMIT_INST\&index=null

Schoepe, H.-J., Bryant, G., \& Van Megen, W. (2006). Two-step crystallization kinetics in colloidal hard-sphere systems. Physical Review Letters, 96(17), 175701-1-175701-175704.

https://doi.org/10.1103/PhysRevLett.96.175701

Published Version: https://doi.org/10.1103/PhysRevLett.96.175701

Repository homepage: https://researchrepository.rmit.edu.au

(c) 2006 The American Physical Society

Downloaded On 2023/04/26 12:15:23 +1000 


\title{
Two-Step Crystallization Kinetics in Colloidal Hard-Sphere Systems
}

\author{
Hans Joachim Schöpe, ${ }^{1}$ Gary Bryant, ${ }^{2}$ and William van Megen $^{2}$ \\ ${ }^{1}$ Institut für Physik, Johannes Gutenberg-Universität Mainz, Staudingerweg 7, D-55128 Mainz, Germany \\ ${ }^{2}$ Department of Applied Physics, RMIT, GPO Box, 2476V, Melbourne 3001, Australia
}

(Received 21 December 2005; published 4 May 2006)

\begin{abstract}
The crystallization kinetics of colloidal hard spheres was studied using a special Bragg spectrometer with high sensitivity. In contrast with the classical scenario we observe a two-step nucleation process: the number of crystallites increases slowly at early times, followed by a dramatic reduction at intermediate times, prior to undergoing a rapid increase at late times. We explain these results in terms of a polydispersity limited growth of crystallites, where the crystallization at early times is governed by local fractionation processes, leading to a long delay prior to final crystallization.
\end{abstract}

DOI: 10.1103/PhysRevLett.96.175701

A complete understanding of the solidification process is one of the long-standing problems in condensed matter physics, and remains of intense research interest (e.g., Ref. [1]). A great deal of progress has been made in recent years using colloidal suspensions as model systems. Because of their large size, both the dynamics and kinetics of these systems are experimentally much more accessible than is the case for atomic and molecular systems.

An important difference between one component atomic systems and (nominally) one component hard-sphere (HS) particles is that colloidal particles always have a particle size distribution (PSD). Recently the effects of polydispersity have attracted a lot of attention from theory and simulation [2], and models of HS systems show that, even for small polydispersities $(<10 \%)$, there is local fractionation or segregation of particle sizes $[3,4]$. This fractionation has also been inferred from experimental studies $[5,6]$, where it has a strong retarding effect on nucleation. These effects are magnified when two components with slightly different sizes are mixed [5-7].

Classical nucleation theory (CNT) assumes that crystallization occurs via a process of nucleation at random sites, after an undercooling dependent delay time, followed by crystal growth. The nucleation process is described by the (time independent) nucleation rate density, which is the number of nuclei appearing per unit time per unit volume (e.g., Ref. [8]). While much of the experimental work on colloidal crystallization is qualitatively consistent with this standard classical view, some observations are not. For example, colloidal crystallization sometimes undergoes multiple stages, rather than a single, simple nucleation process. A two-step process was first observed by Harland [9] for volume fractions near the glass transition, and they speculated that it may be a consequence of nondissipated structures induced by the shear melting process. Similar behavior was observed using Bragg scattering studies in microgravity [10].

Recently, using of an improved spectrometer [11], Martin observed the two-step crystallization process in two systems: particles with an approximately Gaussian PSD at high volume fractions, and for a system with a
PACS numbers: 64.70.Dv, 81.10.Fq, 82.70.Dd

polydispersity skewed to smaller sizes, at all volume fractions [5]. This two-step behavior could be enhanced by artificially skewing the distribution by adding a second component [6]. The conclusion was that the two-step behavior was a consequence of the local fractionation of particles required for crystals to grow, which both slows down crystallization and introduces a delay to the main nucleation process. This work showed the extreme sensitivity of the crystallization kinetics to details of the PSD, and leads to the question: does the two-step process depend on the detailed shape of the polydispersity, or is it a universal feature of polydisperse systems?

Here we present measurements of solidification kinetics for a colloidal HS system with a narrow, very well-defined Gaussian PSD. We calculate for the first time the nucleation rate densities as a function of time, and show that this parameter clearly undergoes a two-step process. We explain these results in terms of limited nucleation at early times, followed by a local fractionation process, prior to the onset of rapid nucleation.

The methods are described in detail elsewhere [5,12] and are briefly summarized here. The particles consist of a core of methylmethacrylate and trifluoroethylacrylate, with a stabilizing coating of poly-12-hydroxystearic acid, about $10 \mathrm{~nm}$ thick. The particles are refractive index matched in the solvent cis-decalin $\left(n=1.483\right.$ at $\left.25^{\circ} \mathrm{C}\right)$. The PSD of the particles was determined by electron microscopy (of $\approx 1000$ particles), dynamic light scattering and static light scattering, and will be reported in detail elsewhere [12]. The results yielded a Gaussian PSD with a polydispersity $\sigma$ of $4.8 \%$ and a hydrodynamic radius of $R_{H}=320 \mathrm{~nm}$, which leads to a Brownian time $t_{\mathrm{B}}=R^{2} / D_{0}=0.5 \mathrm{~s}$, where $D_{0}$ is the free particle diffusion coefficient.

The particles behave as near perfect HS as shown from phase diagrams [13] or direct measurements [14]. The effective HS volume fraction $\phi$ is determined [15] by referencing the observed equilibrium freezing volume fraction $\phi_{\mathrm{f}}$ to the known $\phi_{\mathrm{f}}$ of HS with $\sigma=5 \%$ [4], giving $\phi_{\mathrm{f}}=$ 0.505 and a melting volume fraction of $\phi_{\mathrm{m}}=0.538$. Shear melting of the samples is achieved by tumbling at a frequency of $\approx 1 \mathrm{~Hz}$ for several hours, explained in detail in 
Ref. [5]. Crystallization kinetics were measured using time resolved laser light Bragg scattering, averaging over the whole Debye-Scherrer cone to allow access to data at early times [11]. The angular resolution of the detector is $\Delta \theta=$ $0.02^{\circ}$, giving a resolution around the main peak of about $\Delta q \approx 5 \times 10^{-6} \mathrm{~nm}^{-1}$. Crystal growth is characterized from the measured structure factor $S(q, t)$, as a function of the scattering vector $q$ and elapsed time $t$. The crystalline structure factor $S_{\text {xtal }}$ is extracted from the measured data using the method of Harland [5,16], by subtracting the fluid background $\beta(t) S_{\text {fluid }}$, where the fluid structure factor is obtained at time $t=0$, defined as $60 \mathrm{~s}$ after stopping the shear melting process:

$$
S_{\text {xtal }}(q, t)=S(q, t)-\beta(t) S_{\text {fluid }}(q) .
$$

The parameters extracted from the crystalline structure factor are as follows.

(1) The crystallinity $X(t)$ (the fraction of the sample which is crystalline), determined by integrating the structure factor over the main Bragg reflection, normalized to the equilibrium value:

$$
X(t)=c \int S_{\text {xtal }}(q, t) d q
$$

where $c$ is the normalization factor.

(2) The crystalline volume fraction for a close packed crystal, $\phi_{\text {xtal }}(t)$, determined from the peak maximum, $q_{\max }(t)$, estimated from Gaussian fits to the structure factor peak:

$$
\phi_{\text {xtal }}(t)=\frac{2\left[q_{\max }(t) R\right]^{3}}{9 \sqrt{3} \pi^{2}} .
$$

(3) The average crystal linear dimension, $\langle L(t)\rangle$, determined from the full width at half maximum, $\delta q(t)$ :

$$
\langle L(t)\rangle=\frac{2 \pi K}{\delta q(t)}
$$

where $K=1.107$ is the Scherrer constant for a spherical shaped crystal [17]. From these basic parameters, the following quantities can be determined.

(4) The number of crystals in the scattering volume $V_{\text {scatt }}$ :

$$
N_{\text {xtal }}(t)=\frac{X(t) V_{\text {scatt }}}{\left\langle L^{3}(t)\right\rangle}=\frac{X(t) V_{\text {scatt }}}{\alpha\langle L(t)\rangle^{3}}
$$

where the parameter $\alpha \approx 1.25$ relates the average crystal size cubed, with the average crystal volume (see Ref. [18]).

(5) The nucleation rate density, defined as the rate at which crystals appear in the liquid free volume:

$$
J(t)=\frac{1}{(1-X(t))} \frac{d}{d t} \frac{X(t)}{\left\langle L^{3}(t)\right\rangle} .
$$

This quantity represents the number of critical nuclei which form inside a unit volume of the undercooled liquid. So it is necessary to normalize the nucleation rate with the remaining liquid volume of the sample, rather than the total volume (e.g., Ref. [19]).
Figure 1 shows the time evolution of the crystalline structure factor for the sample with $\Phi=0.538$, broken into three time intervals. Figure 1(a) shows the curves obtained in the first hour following the quench. Here we observe only one broad peak growing slowly in intensity. Between one and two hours after the quench it becomes clear that this peak corresponds to the hcp (002) peak, and the (100) peak begins to grow [Fig. 1(b)], with the (101) becoming clearly visible at later times. The crystallites have now clearly converted from some precursor structure [Fig. 1(a)] into an rhcp structure [Fig. 1(b)]. After the conversion is complete the intensity increases rapidly [Fig. 1(c)], but the structure remains unchanged. We define these three stages of growth, based on the number of crystals, as (a) induction, (b) conversion, and (c) final nucleation. Note that we can be confident that the behavior observed is due to homogeneous nucleation, as previous studies [20] have shown that heterogeneous growth (e.g., from the walls or due to shearing) can be clearly distinguished both spectroscopically and by eye. For the samples presented here there was no evidence of heterogeneous growth. Figure 2 shows the time dependent behavior for a range of parameters extracted from the structure factors shown in Fig. 1: (a) nucleation rate densities $J$, (b) number of crystals $N_{\text {xtal }}$ and crystalline volume fraction $\phi_{\text {xtal }}$, (c) crystallinity $X$ and average crystal size $L$. The bars in (b) correspond to the times for the three different growth stages, as shown in the three frames in Fig. 1.

We now consider the three different growth phases. At early times (the induction period), the amount of nonfluid material is growing slowly while the size of the crystallites

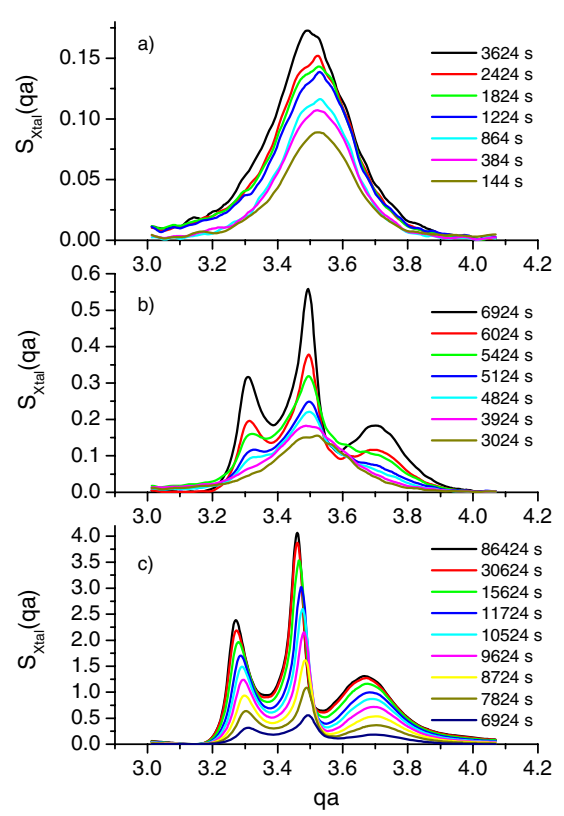

FIG. 1 (color online). Crystalline structure factors at a volume fraction near melting $(\phi=0.538)$ during the three time intervals (a) induction, (b) conversion, and (c) final nucleation. Note the different scales. 


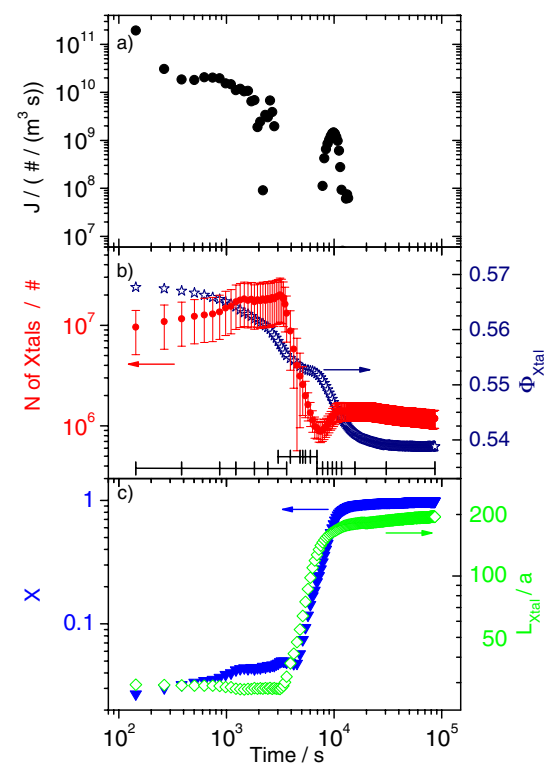

FIG. 2 (color online). Extracted parameters from the data shown in Fig. 1: (a) nucleation rate densities; (b) absolute number of crystals (filled circles, left axis), crystalline volume fraction (open stars, right axis); (c) crystallinity (filled triangles, left axis), average linear dimension (open diamonds, right axis).

stays nearly constant [Fig. 2(c)]. We interpret this as meaning that the number of crystalline precursors in the sample is increasing [Fig. 2(b)], but there is limited growth of these precursors. As a result we find a slowly decreasing nucleation rate density in the first time interval [Fig. 2(a)]. The process begins with dense, compressed precursor crystallites which expand continuously from $\Phi=0.568$ down to $\Phi=0.558$ [Fig. 2(b)]. In the second time interval (the conversion period), the average crystal size grows significantly, to about $65 \%$ of its final maximum value [Fig. 2(c)]. In this interval the crystallinity at first increases slowly, then starts growing rapidly, from 0.05 to 0.14 , but its rapid growth is significantly delayed relative to the crystalline size (by about $1700 \mathrm{~s}$-note the log scale). At the same time the crystal volume fraction [Fig. 2(b)] first decreases rapidly down to 0.552 , then stays nearly constant, and the number of crystals drops by more than 1 order of magnitude [Fig. 2(b)]. This drop in the number of crystals begins at the time when we are first able to identify features of an rhcp structure, i.e., the appearance of the (100) peak in the structure factor [Fig. 1(b)]. In the third time interval (final nucleation), the main nucleation process takes place. Here the crystallinity grows from 0.14 to 1 , while the curve for the average crystal size begins to plateau [Fig. 2(c)]. The crystals expand from $\Phi=0.552$ to $\Phi=0.538$ [Fig. 2(b)]. The absolute number of crystals shows a short sudden increase, becomes constant, and then at long times exhibits a slow drop off [presumably due to ripening - Fig. 2(b)]. The resulting nucleation rate density [Fig. 2(a)] shows a significant increase of more than 1 order of magnitude, and reaches its maximum when the crystallinity is 0.7 , before decreasing to zero.
Clearly, Fig. 2(a) shows that there are two main regions of interest - the induction period at early times, and the final nucleation period at late times. In Fig. 3 we present both the maximum and average values of the nucleation rate densities for each of these periods, as a function of volume fraction (note that the points at the earliest times are ignored due to the experimental uncertainty). As can be seen, the choice of the average or maximum values does not change the shape of these curves. Interestingly, for the induction period, there appears to be only a slight dependence on the degree of undercooling, with a small minimum at the melting volume fraction. For the main nucleation process, on the other hand, we find the expected dependence on undercooling well known from previous experiments $[9,16,21]$ which can be described in a semiquantitative way using CNT. The solid curve shows a two parameter fit ( $A$ and $\gamma$ ) by CNT using the following expression:

$$
\begin{aligned}
J= & A \sqrt{\gamma} D_{s}^{l} \Phi_{\text {xtal }}^{-1 / 3} \Phi_{\text {liq }}^{5 / 3} \\
& \times \exp \left[-4 \pi^{3}(2 a)^{6} \gamma^{3} /\left(3 \Phi_{\text {xtal }}^{2} \Delta \mu^{2} k T\right)\right],
\end{aligned}
$$

where $A$ is a scaling factor, $\gamma$ the fluid-crystal surface tension, $D_{s}^{l}$ the long time self-diffusion coefficient, $\Phi_{\text {xtal }}$ the volume fraction of the crystal, $\Phi_{\text {liq }}$ the volume fraction of the fluid, $a$ the particle radius, and $\Delta \mu$ the chemical potential difference [8]. The resulting dimensionless surface tension $\gamma^{*}=(0.5 \pm 0.05)(2 a)^{2} / k T$ is in good agreement with previous experiments $[9,16,21]$.

Summarizing these results, we find that crystallization does not follow the classical picture of crystal nucleation and growth, but shows three distinct regions.

(1) Induction period: directly following the quench, small, compressed precursors appear which are neither fluid nor true crystals. To interpret these, we turn to real space experiments. Both confocal microscopy [22] and molecular dynamics simulations [23] show that at early times a range of close packed structures occur mixed with other packings (simple or body centered cubic, icosahe-

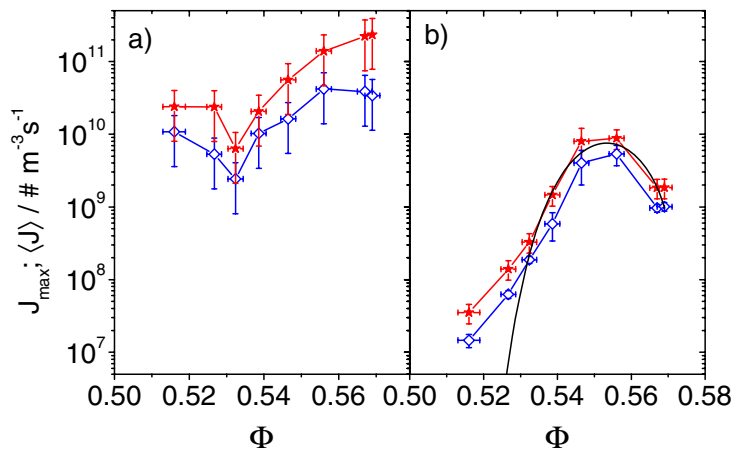

FIG. 3 (color online). Average (open symbols) and maximum (filled symbols) nucleation rate densities as a function of volume fraction. (a) the first crystallization process and (b) the second crystallization process. The solid curve is a fit using CNT with $\gamma^{*}=0.5(2 a)^{2} / k T$. 
dral). Such a mixture of structures could explain the single broad peak observed at early times [Fig. 1(a)]. As the fluid is undercooled, more and more clusters appear, and the peak area grows. Interestingly, the nucleation rate density of these clusters is largely independent of volume fraction [Fig. 3(a)]. The average size of the precursor structures is found to be $\approx 2500$ particles for the example shown. This compares with an estimate of about 1000 particles in a sub critical cluster at $\Phi=0.51$, made using video microscopy [24]. The observed clusters are therefore comparable to or larger than the critical size of a nucleus. However, although these precursors are of the right order to grow, their growth is limited because their structure is not perfect. In order to continue to grow they must first convert into a close packed crystalline structure.

(2) Conversion period: this involves the precursors converting into crystallites with rhcp stacking. When the first true crystallites start to grow, the number of crystallites is decreasing while the crystallinity is still growing. This implies that either the existing precursors merge together into larger crystallites, or that some crystallites are growing at the expense of the remainder, which disappear. In this region the nucleation rate is undefined as the total number of crystals is decreasing [Fig. 1(b)].

(3) Final nucleation: when the average crystal size is close to a maximum and the crystal growth slows, a new accelerated nucleation process takes place. During this interval the growth in crystal size slows, while the crystallinity grows to $\approx 80 \%$. An explanation for this slowing is that the growth of existing crystals is limited by polydispersity [5]: consider a crystalline cluster of particles which are roughly the same size. The further growth of the cluster requires that particles in the surrounding volume join the cluster. There will be a slight preference for particles of the same size, rather than smaller or larger. When the cluster first forms, it will be compressed [i.e., will have a higher volume fraction, as demonstrated in Fig. 2(b)], creating a region of slightly lower density in the surrounding fluid (for discussion see Ref. [7]). The surrounding fluid will therefore have more room for particle rearrangements, so that correctly sized particles are able to join the cluster, while others are excluded. This process will slow the growth of crystallites as they get larger, as more particle rearrangements will be needed.

However, this cannot continue forever-as the cluster grows, it becomes surrounded by a (relatively) high concentration of particles which were previously excluded from the growing crystal lattice. Once the growth of the crystals slows (or stops), more crystals must form elsewhere, as the remaining liquid is still undercooled. This leads to the final nucleation process. The conversion rate is extremely high: while the precursor crystals are converting and growing, the remaining undercooled fluid is undergoing a fractionation process, slowly producing the right conditions for the formation of critical nuclei. Eventually, this reordering has taken place throughout the fluid volume, and the number of crystallites rises dramatically.
The results presented in this Letter imply that the twostep process previously observed may be a universal feature of polydisperse systems, and we predict that it would be observed in any colloidal HS system if the experiments have sufficient sensitivity at early times. This polydispersity dependent induction process is responsible for delaying the onset of primary nucleation. Further, we speculate that the length of the induction process, and the delay time prior to nucleation, are directly related to the amount and type of polydispersity. If the polydispersity is sufficiently small, then the induction time may be too short to be observed.

H. J. S. would like to thank the Alexander von Humboldt Foundation for providing financial support.

[1] M. Asta, F. Spaepen, and J. F. van der Veen, MRS Bull. 29, 920 (2004).

[2] S. Auer and D. Frenkel, Nature (London) 413, 711 (2001); P. Bartlett, J. Phys. Condens. Matter 12, A275 (2000); R. M. L. Evans and C. B. Holmes, Phys. Rev. E 64, 011404 (2001).

[3] P. Bartlett, J. Chem. Phys. 107, 188 (1997).

[4] D. A. Kofke and P. G. Bolhuis, Phys. Rev. E 59, 618 (1999).

[5] S. Martin, G. Bryant, and W. van Megen, Phys. Rev. E 67, 061405 (2003).

[6] S. Martin, G. Bryant, and W. van Megen, Phys. Rev. E 71, 021404 (2005).

[7] S. I. Henderson and W. van Megen, Phys. Rev. Lett. 80, 877 (1998)

[8] B. Mutafschiev, in Handbook of Crystal Growth, edited by D. T. J. Hurle (Elsevier, Amsterdam, 1993), p. 3878.

[9] J.L. Harland et al., Phys. Rev. Lett. 75, 3572 (1995).

[10] Z. Cheng et al., Appl. Opt. 40, 4146 (2001).

[11] P. Francis et al., Rev. Sci. Instrum. 73, 3878 (2002).

[12] H. J. Schöpe, G. Bryant, and W. van Megen (to be published).

[13] P. N. Pusey and W. van Megen, Nature (London) 320, 340 (1986).

[14] G. Bryant et al., Phys. Rev. E 66, 060501(R) (2002).

[15] S. E. Paulin and B. J. Ackerson, Phys. Rev. Lett. 64, 2663 (1990).

[16] J. L. Harland and W. van Megen, Phys. Rev. E 55, 3054 (1997).

[17] A. L. Patterson, Phys. Rev. 56, 978 (1939).

[18] K. M. Dobrich, C. Rau, and C.E. I. Krill, Metall. Mater. Trans. A 35A, 1953 (2004).

[19] V. Erukhimovitch and J. Baram, Phys. Rev. B 50, 5854 (1994).

[20] A. Heymann et al., J. Colloid Interface Sci. 207, 119 (1998); S. Martin, G. Bryant, and W. van Megen, Phys. Rev. Lett. 90, 255702 (2003).

[21] C. Sinn et al., Prog. Colloid Polym. Sci. 118, 266 (2001).

[22] U. Gasser, A. B. Schofield, and D. A. Weitz, J. Phys. Condens. Matter 15, S375 (2003); U. Gasser et al., Science 292, 258 (2001).

[23] B. O’Malley and I. K. Snook, J. Chem. Phys. 123, 054511 (2005).

[24] M.S. Elliot, S. B. Haddon, and W. C. K. Poon, J. Phys. Condens. Matter 13, L533 (2001). 